\title{
Computerised cognitive-behavioural therapy for depression: systematic review
}

\author{
Eva Kaltenthaler, Glenys Parry, Catherine Beverley and Michael Ferriter
}

\section{Background}

Computerised cognitive-behavioural therapy (CCBT) is used for treating depression and provides a potentially useful alternative to therapist cognitive-behavioural therapy (CBT).

\section{Aims}

To systematically review the evidence for the effectiveness of CCBT for the treatment of mild to moderate depression.

\section{Method}

Electronic databases were searched to identify randomised controlled trials. Selected studies were quality assessed and data extracted by two reviewers.

\section{Results}

Four studies of three computer software packages met the inclusion criteria. Comparators were treatment as usual, using a depression education website and an attention placebo.

\section{Conclusions}

There is some evidence to support the effectiveness of CCBT for the treatment of depression. However, all studies were associated with considerable drop-out rates and little evidence was presented regarding participants' preferences and the acceptability of the therapy. More research is needed to determine the place of CCBT in the potential range of treatment options offered to individuals with depression.

\section{Declaration of Interest}

None. Funding detailed in Acknowledgements.
Depression and anxiety are common mental disorders usually treated within a primary care setting in the UK. Recognition of both disorders by general practitioners is often poor and the proportion of individuals who actually receive treatment is low. ${ }^{1}$ Currently, medication is usually the first and often the only treatment offered, but is often associated with unwanted effects and is not cost-effective in those people with negative attitudes to taking medication. ${ }^{2}$ There is substantial evidence to support the use of psychological therapies, particularly cognitive-behavioural therapy (CBT) in the treatment of depression and anxiety. ${ }^{3-5}$ In common with all psychological therapies, there are considerable problems in the delivery of CBT in the UK, including too few therapists, expense associated with service costs, waiting lists and peoples' reluctance to enter therapy. Recently there have been suggestions that self-help strategies are useful tools for delivering psychological therapies. ${ }^{6}$ Alternative delivery methods for CBT with less therapist involvement have been developed including computerised cognitive-behavioural therapy (CCBT). Although a variety of non-randomised uncontrolled studies of CCBT have been reported in the literature, ${ }^{7-9}$ these studies are of poorer quality compared with randomised controlled trials and are potentially biased in favour of the treatment under investigation. This paper systematically reviews randomised controlled trials of CCBT software packages for the treatment of mild to moderate depression.

\section{Method}

The searches aimed to identify all references relating to the clinical effectiveness of CCBT for anxiety and depressive disorders, with particular emphasis on the literature published since the original National Institute for Health and Clinical Excellence (NICE) Guidance 51. ${ }^{10}$ Fifteen electronic bibliographic databases were searched including Medline, Embase, Cochrane and PsycINFO, among others. Population search terms included 'depression AND anxiety' and were combined with intervention terms such as 'cognitive therapy', 'behavio(u)r therapy', 'psychotherapy AND computer', 'computerised', 'internet', etc. These were supplemented with more specific searches on named packages.

In addition, the reference lists of relevant articles were checked and health services research-related resources such as Health Technology Assessment (HTA) organisations, guideline-producing bodies, generic research and trials registers, and specialist mental health sites were also searched. The search strategies and sources are described in detail elsewhere. ${ }^{9}$ No date, language, study or publication type restrictions were applied.

We included studies of adults with mild to moderate depression, with or without anxiety, as defined by individual studies. The following disorders did not fall within the remit of this review: postnatal depression, bipolar disorder, depression with psychotic symptoms or current major depression or serious suicidal thoughts.

Cognitive-behavioural therapy was delivered alone or as part of a package of care either via a computer interface or over the telephone with a computer response. Comparators were current standard treatments including therapist-led CBT, non-directive counselling, primary care counselling, routine management (including drug treatment) and alternative methods of CBT delivery such as bibliotherapy and group CBT. Outcomes included improvement in psychological symptoms, interpersonal and social functioning, quality of life and participant satisfaction both with treatment and site of delivery. All randomised controlled trials (RCTs) meeting the inclusion/exclusion criteria were included.

Quality assessment for the trials was based on the Critical Appraisal Skills Programme ${ }^{11}$ checklist for RCTs. All data from included studies was extracted by one reviewer and checked by a second using a standardised data extraction form.

We assessed studies on the basis of study design, populations, comparators and outcomes for synthesis. Due to heterogeneity in these components, we could not undertake formal statistical synthesis and the results are presented in tabulated format with a narrative synthesis of the results. 


\section{Results}

We screened 455 references and assessed the text of 103 full papers. Figure 1 summarises the study selection and exclusion process. Four studies met the inclusion criteria ${ }^{12-15}$ and details of these studies are presented in online Table DS1. One RCT of Beating the Blues was identified comparing it with treatment as usual (TAU). ${ }^{12}$ One study of MoodGYM had two comparators, a web-based information programme (BluePages) and attention placebo. ${ }^{13}$ Two RCTs of Overcoming Depression on the Internet (ODIN) were identified, with usual care as the comparator. ${ }^{14,15}$ One of these studies ${ }^{15}$ compared two different forms of reminders (telephone or post) in the group receiving ODIN.

\section{Beating the Blues}

Beating the Blues consists of a 15-min introductory video and eight 1-h interactive computer sessions using CBT strategies. Sessions are usually on a weekly basis and completed in the routine care setting (general practice). Homework projects are set and progress reports generated after each session (including reported suicidality). The amount of therapist time reported by Proudfoot et $a l^{12}$ was up to a total of $80 \mathrm{~min}$ over the eight sessions for Beating the Blues.

\section{MoodGYM}

MoodGYM is a web-based CBT programme for depression, developed in Australia. It consists of five interactive modules, which are made available sequentially on a week-by-week basis, with revision of all aspects of the programme in the sixth week. No therapist input was reported in the study identified, ${ }^{13}$ but participants were phoned weekly to ascertain whether the site had been visited, to encourage repeat visits and homework completion.

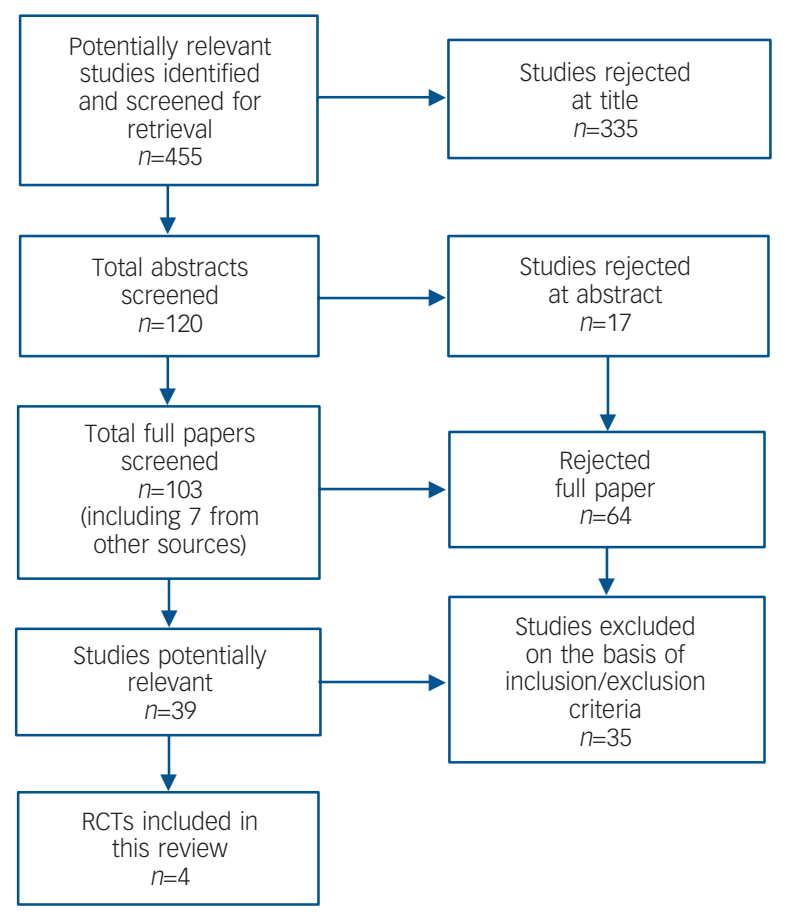

Fig. 1 Summary of study selection and exclusion. RCTs, randomised controlled trials.

\section{Overcoming Depression on the Internet}

Overcoming Depression on the Internet, a US-based programme, uses cognitive restructuring techniques delivered over the internet in the form of self-guided interactive tutorials. The ODIN site is unattended, and there is no therapist involvement. However, participants in the telephone reminder group were phoned by study staff who had non-clinical backgrounds. ${ }^{15}$ Participants were free to sign in and use the site as desired. In one study ${ }^{14}$ the mean number of sessions was 2.6 (s.d. $=3.5$; range $1-20$ sessions). Sessions were self-paced and the length of the sessions was not reported in either study. In the second study (with reminders by post or telephone) the mean number of sessions was 5.9 (s.d. $=6.2$; range $1-33$ sessions) for reminders by post and 5.6 (s.d.=5.8; range $1-27$ sessions) for reminders by telephone. ${ }^{15}$

\section{Study characteristics}

All four studies had mostly female participants with mean age varying from 36.43 years $(\text { s.d. }=9.4)^{13}$ to 50.3 years (s.d.=10.8). ${ }^{15}$ Methods for diagnosis of depression varied. Proudfoot et $_{\text {al }}{ }^{12}$ used the Programmable Questionnaire system ${ }^{16}$ to diagnose depression, while Christensen et al $^{13}$ used the Kessler Psychological Distress Scale $^{17}$ and Clarke et $a l^{14,15}$ identified participants who had received medical services in the previous 30 days with a recorded diagnosis of depression. Both ODIN studies included participants with and without depression.

\section{Study quality}

The quality of the studies was moderate, as briefly described in online Table DS1. However, two studies reported no reasons for loss to follow-up ${ }^{14,15}$ and none of the four studies used masked assessment of outcomes. Data on psychological outcomes and participant satisfaction for the four studies are presented in online Tables DS2-DS5.

\section{Psychological outcomes}

The four studies used a variety of instruments to measure psychological outcomes, making comparison between the trials difficult. The Beating the Blues study ${ }^{12}$ used the Beck Depression Inventory ${ }^{18}$ as the primary outcome measure. Beating the Blues appeared to be more effective than TAU with regard to scores for depression.

In the MoodGYM study, ${ }^{13}$ the Centre for Epidemiologic Studies Depression (CES-D) Scale ${ }^{19}$ was used as the primary outcome measure. Mean improvement in symptoms was reported rather than pre- and post-treatment values. Both MoodGYM (CCBT) and BluePages (psychoeducation) delivered via the internet were more effective in reducing symptoms of depression than the control. The two ODIN trials also used CES-D as the primary outcome measure. In one of the ODIN studies, ${ }^{14}$ ODIN was not effective in reducing symptoms of depression. In the other ODIN trial, ${ }^{15}$ participants had a greater reduction on the CES-D than those in the TAU control group.

All four studies reported the use of an intention-to-treat analysis. However, Proudfoot et $\mathrm{al}^{12}$ reported pre-treatment values only for 127 of the 146 randomised participants in the Beating the Blues group and for 114 of the 128 individuals in the TAU group making this not strictly speaking an intention-to-treat analysis.

\section{Participant satisfaction}

The four studies gave some, although limited, information on participants' attitudes to treatment. Proudfoot et $a l^{12}$ reported that individuals in the Beating the Blues group were significantly 
more satisfied with treatment than those in the TAU group but no values were reported. Christensen et al ${ }^{13}$ reported that peoples' acceptability of MoodGYM was implied by the low drop-out rates although no data were reported. No information regarding participants attitudes to treatment was reported for either ODIN trial.

\section{Drop-out rates}

Drop-out rates varied between the studies. In the study comparing Beating the Blues with $\mathrm{TAU},{ }^{12}$ of the 274 individuals initially randomised, 35\% dropped out during the study. In the MoodGYM study, ${ }^{13} 25.3 \%$ dropped out of the MoodGYM group, while $15 \%$ dropped out of the BluePages group and $10 \%$ from the attention placebo group. In the ODIN trials it is difficult to differentiate drop-out rates in the treatment group from the control groups as overall drop-out numbers are reported. In one ODIN trial, of the 299 participants initially randomised, $26 \%$ failed to complete at least one follow-up visit and by the 32-week followup the drop-out rate had reached $41 \% .{ }^{14}$ In the other ODIN study, $18 \%$ of the initial 255 participants failed to complete at least one follow-up assessment and at 16 weeks this had reached $34 \% .{ }^{15}$ These drop-out rates are comparable with those for other psychological therapies for depression. ${ }^{20}$

\section{Discussion}

Four RCTs were identified in this systematic review of CCBT for treatment of mild to moderate depression, three of which show evidence of effectiveness. There is some evidence that Beating the Blues is more effective than treatment as usual for depression. Both MoodGYM (CCBT) and BluePages (psychoeducation) delivered via the internet were effective in reducing symptoms of depression. With regard to the ODIN studies, one study ${ }^{14}$ reported no treatment effect for ODIN and low usage rates in the ODIN intervention group. In the second study, ${ }^{15}$ intervention participants had a greater reduction in depression scores compared with the control group. There is limited evidence from all of the studies regarding the satisfaction of participants.

Although CCBT is a potentially useful intervention, the identified trials were of moderate quality and associated with several weaknesses. For example, comparisons between the packages are difficult owing to the use of different comparators and outcome measures, and different levels of severity at baseline. It is also difficult to ascertain the amount of therapist involvement needed by individuals using the programmes. There is a lack of independent studies, with most trials being conducted by those developing and promoting the products.

Implementation of programmes may be hindered by lack of information regarding recruitment. In most of the included studies the individuals were self-selected, in that they volunteered to take part in the study. In routine primary care, not all individuals may be willing to try CCBT. Little information was provided by Proudfoot et al ${ }^{12}$ regarding the routine selection of participants in general practitioner surgeries.

\section{Methodological strengths and weaknesses}

The studies reported in this systematic review are moderate quality RCTs, which by virtue of their design attempt to reduce potential biases. However, the studies used self-selected individuals and there is very little data on those who refused to participate or those who dropped out. By including only RCTs we may have missed important data from non-randomised comparative trials. Randomised controlled trial results can be supplemented by comparative outcome data from large patient-population field trials to ascertain the extent to which results obtained in efficacy trials can be translated into clinically effective interventions within complex healthcare systems. Other limitations are that the session duration was not always specified as well as the method of participant selection and severity of depressionmaking comparisons between trials difficult. Any extension of these findings to other groups, including those with severe depression, must be made with caution.

\section{Areas for further research}

Future trials should include appropriate comparators such as bibliotherapy or selective serotonin reuptake inhibitors and study design should incorporate qualitative data collection regarding individual preference and attitudes towards treatment. Information is also needed to determine why individuals drop out of treatment. Pragmatic trials of CCBT within a stepped care programme are also necessary.

Increasingly, CCBT is offered as part of a range of treatments for people with depression, especially where access to CBT is limited. However, there is no evidence to suggest that all those would wish to participate in treatment via a computer interface.

People with depression at present have relatively few treatment options. Computerised CBT potentially allows treatment to be offered to a far greater number of individuals than is currently possible and offers considerable savings in terms of therapist time to a subset of those with depression.

Eva Kaltenthaler, BSC, MSC, PhD, Glenys Parry, BA, PhD, DipClinPsych, CPsycho FBPSS, Catherine Beverley, MSC, BSC, MCLIP, School of Health and Related Research (ScHARR), University of Sheffield; Michael Ferriter, BA, MPhil, MSC, PhD, Department of Research and Development Nottinghamshire Healthcare NHS Trust, Rampton Hospital, Nottingham, UK

Correspondence: Eva Kaltenthaler, ScHARR, University of Sheffield, Regent Court, 30 Regent Street, Sheffield S1 4DA, UK. Email: e.kaltenthaler@ sheffield.ac.uk

First received 4 May 2006, final revision 28 Feb 2008, accepted 18 Mar 2008

\section{Acknowledgements}

This project was funded by the NIHR Health Technology Assessment Programme (project number 04/01/04) and commissioned on behalf of NICE. It has been published in full in Health Technology Assessment, Vol. 10, No. 33. See the HTA Programme website (www.hta.ac.uk) for further project information. The views and opinions expressed therein are those of the authors and do not necessarily reflect those of the Department of Health.

\section{References}

1 National Health Service Centre for Reviews and Dissemination. Improving the recognition and management of depression in primary care. Eff Health Care 2002; 7: 1-11.

2 Pyne JM, Rost KM, Farahati F, Tripathi SP, Smith J, Williams DK, Fortney J, Coyne JC. One size fits some: the impact of patient treatment attitudes on the cost-effectiveness of a depression primary-care intervention. Psychol Med 2005; 35: 839-54

3 Department of Health. Treatment Choice in Psychological Therapies and Counselling. Evidence Based Clinical Practice Guideline. Department of Health, 2001.

4 National Institute for Health and Clinical Excellence. Clinical Guidelines for the Management of Panic Disorder and Generalised Anxiety Disorder. NICE, 2004 (http://www.nice.org.uk/page.aspx?0=235400).

5 National Institute for Health and Clinical Excellence \& National Collaborating Centre for Mental Health. Depression: Management of Depression in Primary and Secondary Care. NICE, 2004 (http://www.nice.org.uk/ cg023NICEguideline)

6 Richards DA, Lovell K, McEvoy P. Access and effectiveness in psychological therapies: self-help as a routine health technology. Health Soc Care Community 2003; 11: 175-82. 
7 Kaltenthaler E, Brazier JE, de Nigris E, Tumur I, Ferriter M, Beverley C, Parry G, Rooney G, Sutcliffe P. Computerised cognitive behaviour therapy for depression and anxiety update: a systematic review and economic evaluation. Health Technol Assess 2006; 10: 1-186.

8 Kaltenthaler E, Parry G, and Beverley C. Computerized cognitive behaviour therapy: a systematic review. Behav Cogn Psychother 2004; 32: 31-55.

9 Kaltenthaler E, Shackley P, Stevens K, Beverley C, Parry G, Chilcott J. A systematic review and economic evaluation of computerised cognitive behaviour therapy for depression and anxiety. Health Technol Assess 2002 6: $1-89$

10 National Institute for Health and Clinical Excellence. Guidance on the Use of Computerised Cognitive Behavioural Therapy for Anxiety and Depression. NICE, 2002

11 Critical Appraisal Skills Programme. Checklist for Appraising RCTs. CASP, 2004 (http://www.phru.nhs.uk/Pages/PHD/resources.htm).

12 Proudfoot J, Ryden C, Everitt B, Sharpio DA, Goldberg D, Mann A, Tylee A, Marks I, Gray JA. Clinical efficacy of computerised cognitive-behavioural therapy for anxiety and depression in primary care: randomised controlled trial. Br J Psychiatry 2004; 185: 46-54.

13 Christensen $\mathrm{H}$, Griffiths KM, Jorm AF. Delivering interventions for depression by using the internet: randomised controlled trial. BMJ 2004; 328: 265
14 Clarke G, Reid E, Eubanks D, O'connor E, DeBar LL, Kelleter C, Lynch F, Nunley $\mathrm{S}$. Overcoming depression on the Internet (ODIN): a randomized controlled trial of an Internet depression skills intervention program. $J$ Med Internet Res 2002; 4: e14.

15 Clarke G, Eubanks D, Reid E, Kelleter C, O'Connor E, DeBar LL, Lynch F, Nunley S, Gullion C. Overcoming Depression on the Internet (ODIN) (2): a randomized trial of a self-help depression skills program with reminders. J Med Internet Res 2005; 7: e16.

16 Lewis G. Assessing psychiatric disorder with a human interviewer or a computer. J Epidemiol Community Health 1994; 48: 207-10.

17 Andrews G, Slade T. Interpreting scores on the Kessler Psychological Distress Scale (K10). Aust N Z J Public Health 2001; 25: 494-7.

18 Beck AT, Steer A, Brown GK. Beck Depression Inventory Manual (2nd edn) Psychological Corporation, 1996.

19 Randolf LS. The CES-D Scale: a self-report depression scale for research in the general population. Appl Psychol Meas 1977; 1: 385-401.

20 Kaltenthaler E, Sutcliffe P, Parry G, Beverley C, Rees A, Ferriter M. The acceptability to patients of computerized cognitive behaviour therapy for depression: a systematic review. Psychol Med 2008; 21: 1-10.

EXTRA 